\author{
Nguyen Cao Don \\ Nguyen Thi Minh Hang \\ Hiroyuki Araki \\ Hiroyuki Yamanishi \\ Kenichi Koga
}

\section{Groundwater resources management under environmental constraints in Shiroishi of Saga plain, Japan}

Published online: 2 February 2006

(C) Springer-Verlag 2006

The online version of the original article can be found at http://dx.doi.org/10.1007/ s00254-005-0109-9

N. C. Don

Hydraulic Engineering Consultants

I (HEC-1), Dong Da, Hanoi, Vietnam

N. T. M. Hang

Graduate School of Science

and Engineering, Saga University, Honjo 1,

Saga-shi, 840-8502 Saga, Japan

H. Araki · H. Yamanishi · N. C. Don ( $₫)$ Institute of Lowland Technology,

Saga University, Honjo 1, Saga-shi,

840-8502 Saga, Japan

E-mail: don@ilt.saga-u.ac.jp

Tel.: + 81-952-288571

Fax: + 81-952-288571

K. Koga

Department of Civil Engineering,

Saga University, Honjo 1, Saga-shi,

840-8502 Saga, Japan

\section{Environ Geol (2005). DOI: 10.1007/ s00254-005-0109-9}

The corresponding author Nguyen Cao Don currently belongs to:

Institute of Lowland Technology, Saga University, Honjo 1, Saga-shi, Saga 840-8502, Japan e-mail: don@ilt.saga-u.ac.jp

Tel.: + 81-952-288571

Fax: + 81-952-288571 and previously worked for:

Hydraulic Engineering Consultants I (HEC-1), Dong Da, Hanoi, Vietnam. 